\title{
Substrate stiffness-dependent carbon nanotubes-induced lung fibrogenesis
}

Kai Wang ${ }^{1}$, Lin Shi ${ }^{1}$, Will Linthicum ${ }^{2}$, Kun Man ${ }^{1}$, Xiaoqing $\mathrm{He}^{3}$, Qi Wen ${ }^{2,4}$, Liying Wang Rojanasakul, Yon Rojanasakul ${ }^{3}$, Yong Yang ${ }^{1 *}$

1. Department of Biomedical Engineering, University of North Texas, Denton, Texas, USA 76207

2. Department of Biomedical Engineering, Worcester Polytechnic Institute, Worcester, Massachusetts, USA 01609

3. Department of Pharmaceutical Sciences, West Virginia University, Morgantown, West Virginia, USA 26506

4. Department of Physics, Worcester Polytechnic Institute, Worcester, Massachusetts, USA 01609

5. Allergy and Clinical Immunology Branch, National Institute for Occupational Safety and Health, Morgantown, West Virginia, USA 26505

*Correspondence: Yong Yang (yong.yang@unt.edu), K220, Discovery Park, 3940 N Elm St., Denton, Texas, 76207. Tel: +1-940-565-2982, Fax: +1-940-369-8570 


\section{Materials and Methods}

\section{Preparation of PAAm hydrogels}

PAAm hydrogels were prepared according to the published protocols. ${ }^{1-3}$ Briefly, glass coverslips were first activated by treating with $0.1 \mathrm{~N}$ sodium hydroxide (Sigma-Aldrich, St. Louis, MO, USA) for 3 min, $0.5 \%$ 3-aminopropyltrimethoxysilane (Sigma-Aldrich) for $30 \mathrm{~min}$ and then $0.5 \%$ glutaraldehyde (Fisher Scientific, Hanover Park, IL, USA) for $30 \mathrm{~min}$. By varying the amount of acrylamide (Fisher Scientific) and bis-acrylamide (Fisher Scientific), PAAm hydrogels with the stiffness of $5 \mathrm{kPa}, 21 \mathrm{kPa}$ and $100 \mathrm{kPa}$ were prepared on the activated glass coverslips. The gel stiffness was measured by using the atomic force microscopy (AFM). ${ }^{3}$ Details regarding the amount of each gel components and the measured gel stiffness were provided in Table S1. To facilitate the cell adhesion, fibronectin $(100 \mu \mathrm{g} / \mathrm{mL}$, Corning, Tewksbury, MA, USA) was conjugated on the PAAm hydrogel surfaces by using sulfo-SANPAH (Thermo Fisher Scientific, Carlsbad, CA, USA) as the crosslinker. ${ }^{3}$

\section{Dispersion of MWCNTs}

The MWCNTs (XNRI MWNT-7, Mitsui \& Company, NY, USA) dispersion was prepared as previously reported. ${ }^{4}$ Briefly, $1 \mathrm{mg}$ of MWCNTs was dispersed in $1 \mathrm{~mL}$ of phosphate buffered saline (PBS, Life Technologies, Eugene, OR, USA) containing $5 \mathrm{mg}$ of bovine serum albumin (BSA, Sigma-Aldrich) by sonicating in a biological safety hood. The MWCNT stock solution was stored at $4{ }^{\circ} \mathrm{C}$ for long-term use. Before added to the cells, the stock solution was first sonicated to redisperse MWCNTs evenly, and then diluted in the culture medium to obtain the desired concentrations $(0.3-3 \mu \mathrm{g} / \mathrm{mL})$.

\section{Primary cell culture}

Normal human lung fibroblasts (NHLFs, Lonza, Walkersville, MD, USA, Catalog \#: CC-2512) were primary cells and cultured in the fibroblast basal medium (Lonza) supplemented with 2\% FBS, 0.1\% recombinant human fibroblast growth factor basic (rhFGF-B), $\quad 0.1 \%$ insulin, $\quad 0.1 \%$ 
gentamicin/amphotericin-B, $100 \mathrm{U} / \mathrm{ml}$ penicillin and $100 \mu \mathrm{g} / \mathrm{ml}$ streptomycin. Cells of passages 3-6 were used in this study.

\section{Cellular uptake of MWCNTs}

The cellular uptake of MWCNTs was quantified by applying the turbidimetric method as previously published. ${ }^{5}$ Briefly, NHLFs cultured on PAAm gels or TCPS were treated with $1 \mu \mathrm{g} / \mathrm{mL}$ of MWCNTs for three days, then washed with PBS and lysed in radioimmune precipitation assay (RIPA) buffer (Thermo Fisher Scientific) containing protease inhibitor (Thermo Fisher Scientific) for 30 min on ice. Sonication was applied to disperse the MWCNTs evenly in the cell lysate. The optical density (O.D.) value of cell lysate at $640 \mathrm{~nm}$ was then measured and used to calculate the concentration of MWCNTs based on the standard curve (Figure S6). The total protein concentration was measured by using the BCA protein assay kit (Thermo Fisher Scientific) based on the manufacturer's protocol. The amount of MWCNTs inside the cells was calculated by normalizing the MWCNT concentration in cell lysate to the total protein concentration.

\section{Cell proliferation}

To evaluate the influence of MWCNT dosage on cell proliferation, NHLFs were seeded in the 96well plates at the density of 5000 cells per well and allowed to attach overnight. The cells were then treated with MWCNTs at different concentration $(0.3-3 \mu \mathrm{g} / \mathrm{mL})$ for $1,2,3$ and 4 days. For each group, three parallel samples were prepared. After the designated days of MWCNT treatment, cell proliferation was tested by using CellTiter 96® AQueous One Solution Cell Proliferation kit (Promega, Madison, WI, USA) according to the manufacturer protocol. Briefly, the CellTiter 96® AQueous One Solution Reagent (1:5 dilution in fibroblast growth media) was added to each well and incubated with the cells for $4 \mathrm{~h}$ at $37{ }^{\circ} \mathrm{C}$. The absorbance of each well at the wavelength of $490 \mathrm{~nm}$ was then measured using a FLUOstar OPTIMA Microplate Reader (BMG LABTECH, Cary, NC, USA). 
To assess the cell proliferation on the substrates of different stiffness, the NHLFs were seeded in 48-well plates (TCPS) and on PAAm gels $(5 \mathrm{kPa}$ and $100 \mathrm{kPa})$ that were supported on $8 \mathrm{~mm}$ glass coverslips, which fitted into 48-well plates. For each group, three parallel samples were prepared. After 3 days of MWCNT treatment the PAAm gels with cells were transferred to new 48-well plates, and incubated with the diluted CellTiter 96® AQueous One Solution Reagent. For TCPS samples, the original media was replaced by diluted CellTiter $96 \AA$ AQueous One Solution Reagent. All plates were then incubated for an additional $4 \mathrm{~h}$ at $37^{\circ} \mathrm{C}$. After incubation, $200 \mu \mathrm{L}$ solution was pipetted into a 96-well plate. The absorbance of each well at the wavelength of $490 \mathrm{~nm}$ was then measured. The data on TCPS was used as a control. The culture medium containing the reagent was set as background.

\section{Immunofluorescent staining}

The cells were fixed with 4\% paraformaldehyde (PFA, Sigma-Aldrich) for $30 \mathrm{~min}$ at room temperature, permeabilized and blocked using a PBS solution containing $0.03 \mathrm{~g} / \mathrm{mL} \mathrm{BSA,} 1 \%$ goat serum

(Gibco, Grand Island, NY, USA), and 0.2\% Triton-X 100 (Sigma-Aldrich) for $1 \mathrm{~h}$. The samples were incubated with primary antibody (in PBS solution with $0.2 \%$ Triton-X 100) for $2 \mathrm{~h}$, and then incubated with secondary antibody for $1 \mathrm{~h}$, both at room temperature. Nuclei were counterstained and mounted using ProLong Gold Antifade Reagent with DAPI (Life Technologies). F-actin was stained with Alexa Fluor 488 phalloidin (Life Technologies). Details regarding the antibodies information and dilution were listed in Table S2. The fluorescent images were taken by using a Nikon Ti Eclipse Fluorescence Microscope. The confocal images were taken by using the Zeiss Violet Confocal Microscope.

\section{Reactive oxygen species (ROS) detection}

NHLFs were cultured overnight and then treated with $1 \mu \mathrm{g} / \mathrm{mL}$ MWCNTs for $16 \mathrm{~h}$. The culture medium was then replaced with $1 \mathrm{~mL}$ of Hank's balanced salt solution (HBSS, Sigma Aldrich) containing $10 \mu \mathrm{M}$ dichlorodihydrofluorescein diacetate (DCFDA, Thermo Fisher Scientific) and incubated for an additional $30 \mathrm{~min}$. The cells were finally fixed using 4\% PFA solution and the nuclei were visualized using 
ProLong Gold Antifade Reagent with DAPI. At least 10 images for each group were taken under the Nikon Ti Eclipse fluorescence microscope with the same setting. The fluorescence intensities of images were analyzed by using ImageJ (https://imagej.nih.gov/ij/), and the ROS production per cell was calculated by subtracting the background fluorescence intensity from total image fluorescence intensity, then divided by the cell number. ${ }^{4}$

\section{Western blot analysis}

The cells were lysed by using RIPA buffer containing protease inhibitor for $30 \mathrm{~min}$ on ice, and the obtained proteins were separated by $10 \%$ sodium dodecyl sulfate-polyacrylamide gel electrophoresis (SDSPAGE) and then transferred to polyvinylidene difluoride (PVDF) membranes (EMD Millipore, Burlington, MA, USA). The PVDF membranes were blocked with 5\% nonfat milk in $1 \times$ Tris-buffered saline with $0.1 \%$ Tween-20 (TBST) for $1 \mathrm{~h}$ at room temperature, blotted with the primary antibodies at $4{ }^{\circ} \mathrm{C}$ overnight, and then incubated with horseradish peroxidase-conjugated secondary antibodies (Jackson Immunoresearch Laboratories, Inc) for $1 \mathrm{~h}$ at room temperature. Details regarding the antibodies information and dilution were listed in Table S2. Protein bands were visualized using enhanced chemiluminescence detection reagents (EMD Millipore) and acquired by using a C DiGit Blot Scanner (LICOR Biosciences, Lincoln, NE, USA). The quantification of protein band densitometry was analyzed by using ImageJ software.

\section{FAK and ROCK inhibition}

FAK Inhibitor 14 (Sigma-Aldrich) was used to inhibit the FAK expression and phosphorylation. Briefly, NHLFs were allowed to attach on $100 \mathrm{kPa}$ hydrogels overnight, then treated with FAK Inhibitor $14(5 \mu \mathrm{M})$. MWCNTs $(1 . \mu \mathrm{g} / \mathrm{mL})$ were added $2 \mathrm{~h}$ after FAK Inhibitor 14 treatment. Then the cells were cultured for three days in the presence of both MWCNTs and FAK Inhibitor 14. 
Y27632 (Sigma-Aldrich) was used to reduce the intracellular tension of NHLFs. After cells were seeded and attached overnight, the Y27632 was added to cell culture media $2 \mathrm{~h}$ before MWCNTs treatment at the final concentration of $10 \mu \mathrm{M}$.

\section{Statistical analysis}

The data were presented as mean \pm standard error of the mean (S.E.M.). GPower software (Version 3.1) was used to determine the sample size. In the software, the $\alpha$ and power $(1-\beta)$ value were set as 0.05 and 0.95 , respectively, while the effect size was calculated based on the mean for each group and an estimated standard deviation for all groups. The statistical significances for all the results were analyzed based on one-way analysis of variance (ANOVA) followed by Tukey's HSD (honestly significant difference) test by using JMP software (version 14.1). Statistically significant difference was considered at a level of $p<0.05$. 
Table S1. Preparation and characterization of PAAm hydrogels

\begin{tabular}{|c|c|c|c|c|c|c|}
\hline PAAm & $\begin{array}{c}\mathbf{4 0 \%} \\
\text { Acrylamide } \\
(\boldsymbol{\mu L})\end{array}$ & $\begin{array}{c}\mathbf{2 \%} \text { Bis- } \\
\text { acrylamide } \\
(\boldsymbol{\mu L})\end{array}$ & $\begin{array}{c}\mathbf{5 0 m M} \\
\text { HEPES }(\boldsymbol{\mu} \mathbf{L}) \\
(\mathbf{p H ~ 8 . 2})\end{array}$ & $\begin{array}{c}\text { TEMED } \\
(\boldsymbol{\mu} \mathrm{L})\end{array}$ & $\begin{array}{c}\mathbf{1 0 \%} \\
\text { Ammonium } \\
\text { Persulfate }(\boldsymbol{\mu} \mathrm{L})\end{array}$ & $\begin{array}{c}\text { Measured } \\
\text { Stiffness } \\
(\mathbf{k P a})\end{array}$ \\
\hline $\mathbf{5} \mathbf{~ k P a}$ & 62.5 & 25 & 412.5 & 1.5 & 5 & $4.9 \pm 0.5$ \\
\hline $\mathbf{2 1} \mathbf{~ k P a}$ & 150 & 25 & 325 & 1.5 & 5 & $21.2 \pm 0.5$ \\
\hline $\mathbf{1 0 0} \mathbf{~ k P a}$ & 150 & 125 & 225 & 1.5 & 5 & $100.8 \pm 2.1$ \\
\hline
\end{tabular}

*: The Young's modulus of PAAm gels was measured by using the atomic force microscopy.

Table S2. Information and dilution of antibodies

\begin{tabular}{|l|l|l|l|}
\hline Primary Antibodies & Vendor & Catalog \# & Dilution \\
\hline YAP & Santa Cruz & sc-101199 & $1: 200(\mathrm{IF})$ \\
\hline Collagen I* & Fitzgerald & 70 R-CR007x & $1: 3000(\mathrm{WB})$ \\
& Thermo Fisher Scientific & PA1-26204 & $1: 3000(\mathrm{WB})$ \\
\hline FAK & Santa Cruz & sc-932 & $1: 1000(\mathrm{WB})$ \\
\hline pFAK & Santa Cruz & sc-81493 & $1: 1000(\mathrm{WB})$ \\
\hline$\alpha$-Tubulin & Sigma-Aldrich & T9026 & $1: 2000(\mathrm{WB})$ \\
\hline F-actin & Thermo Fisher Scientific & MA1-80729 & $1: 500(\mathrm{WB})$ \\
\hline
\end{tabular}

*: Collagen I primary antibodies were supplied from Fitzgerald (Figure 2c, 3c and 4f) and Thermo Fisher Scientific (Figure 4b). 

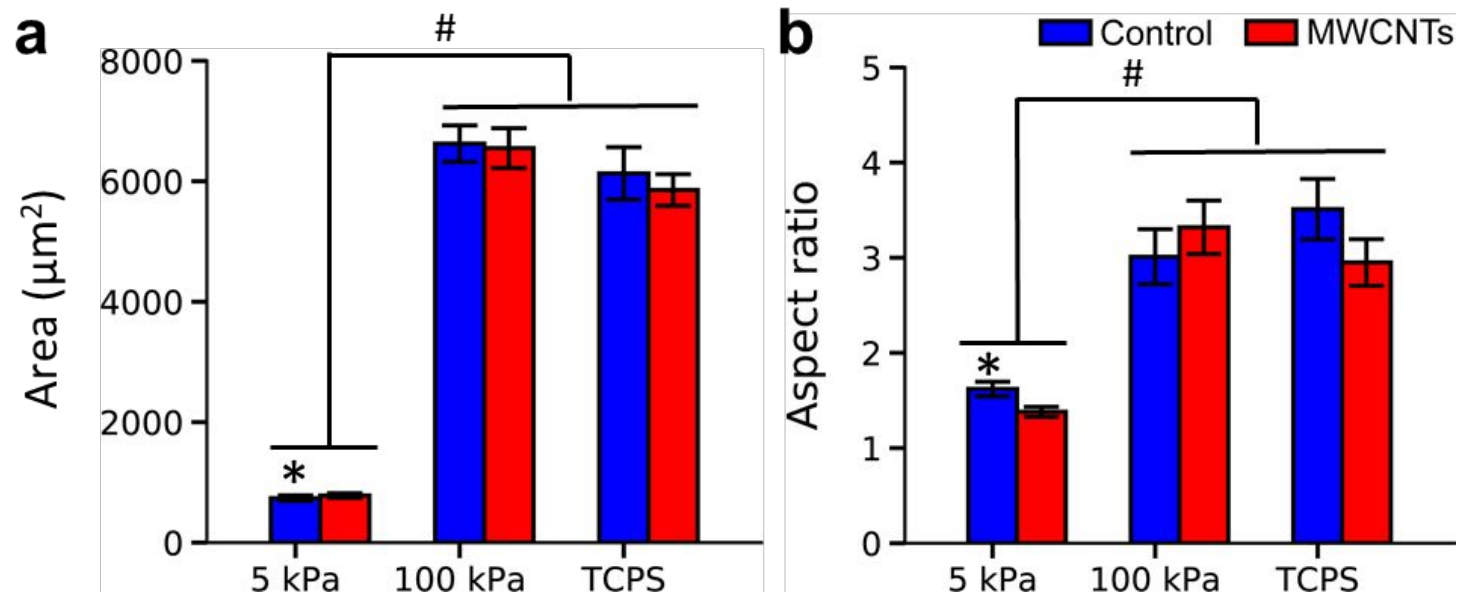

Figure S1: Influence of substrate stiffness on the cell area (a) and aspect ratio (b) of NHLFs with or without MWCNTs treatment. *: significant difference $(p<0.05)$ from the TCPS controls (no MWCNTs treatment); \#: significant difference $(p<0.05)$ between groups. $\mathrm{n}>50$ for all groups.

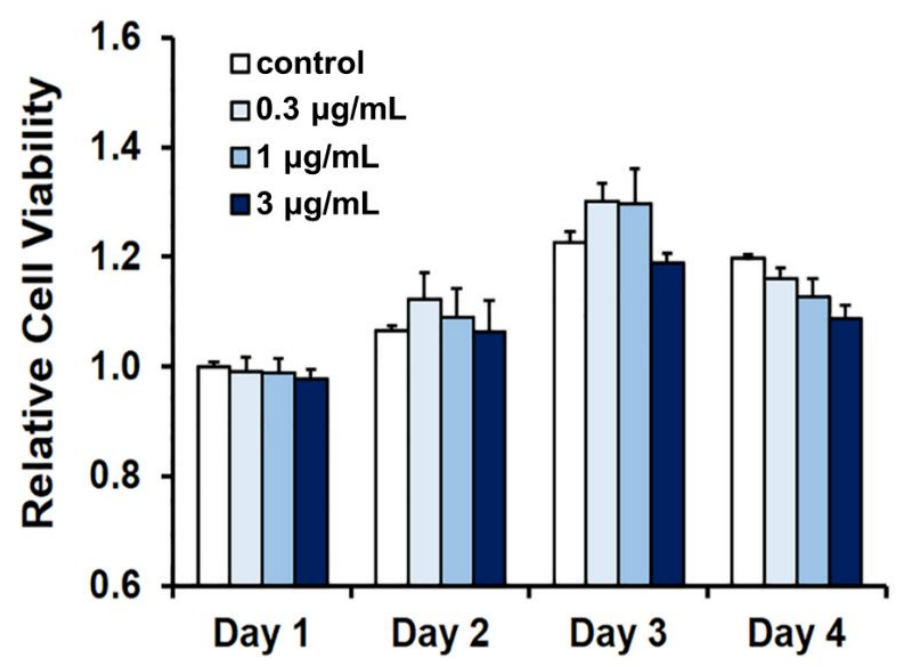

Figure S2. Influence of MWCNTs dosage on the proliferation of NHLFs. All the data were normalized to the mean value of Day 1 cell viability data in the control group (no MWNCTs treatment). $n=3$. 


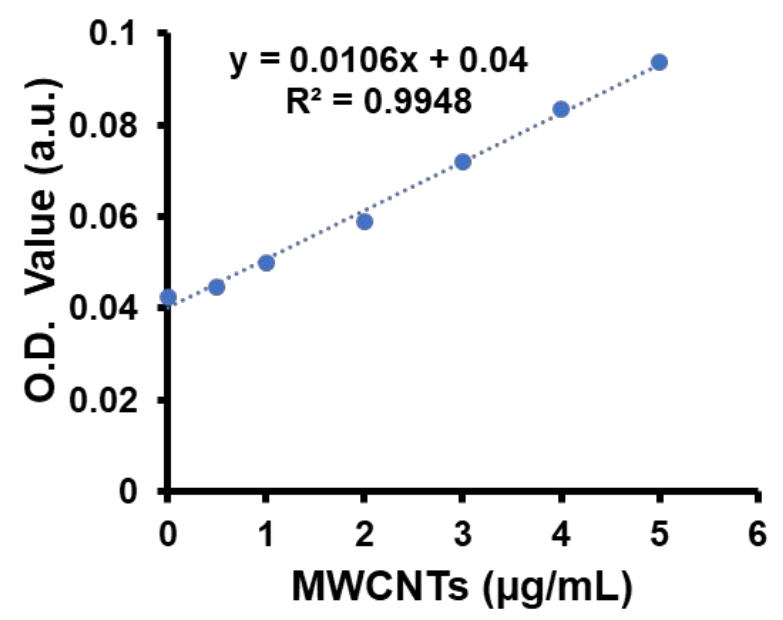

Figure S3. Standard curve of O.D. (640 nm) value of MWCNTs at different concentrations.

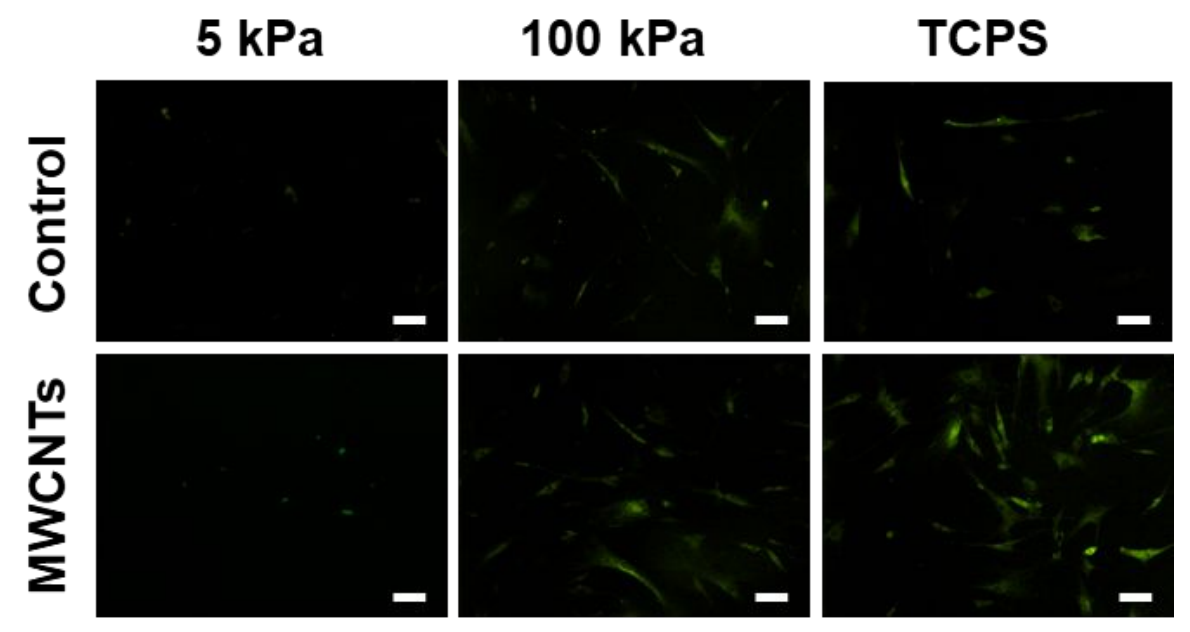

Figure S4. Fluorescent images of NHLFs on different substrates with the staining of DCFDA to detect the generated ROS inside the cells with or without MWCNTs treatment. Green: ROS. Scale bars are $200 \mu \mathrm{m}$. 

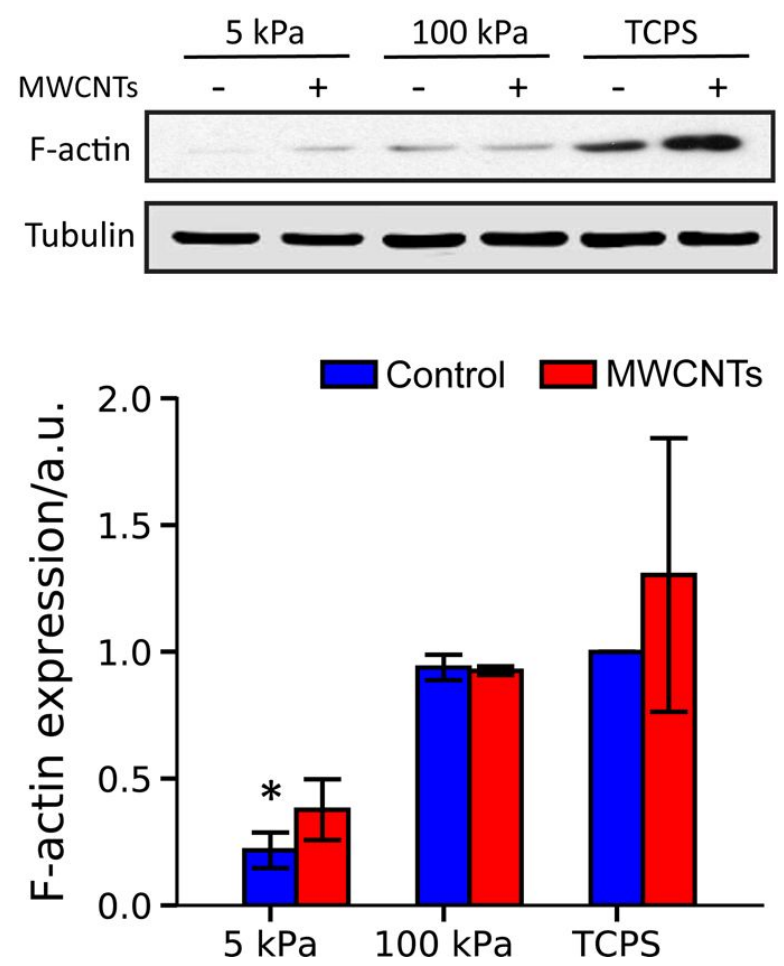

Figure S5. Western blot analysis of the F-actin expression in NHLFs on different substrates with or without MWCNTs treatment. Control: without MWCNTs treatment. MWCNTs: with MWCNTs treatment. Tubulin was used as the loading control. All the data were normalized to the mean value of the TCPS group without MWCNTs treatment. Negative and positive signs indicated the cells were treated without and with MWCNTs, respectively. *: significant difference $(p<0.05)$ from the TCPS control. $\mathrm{n}=3$ 

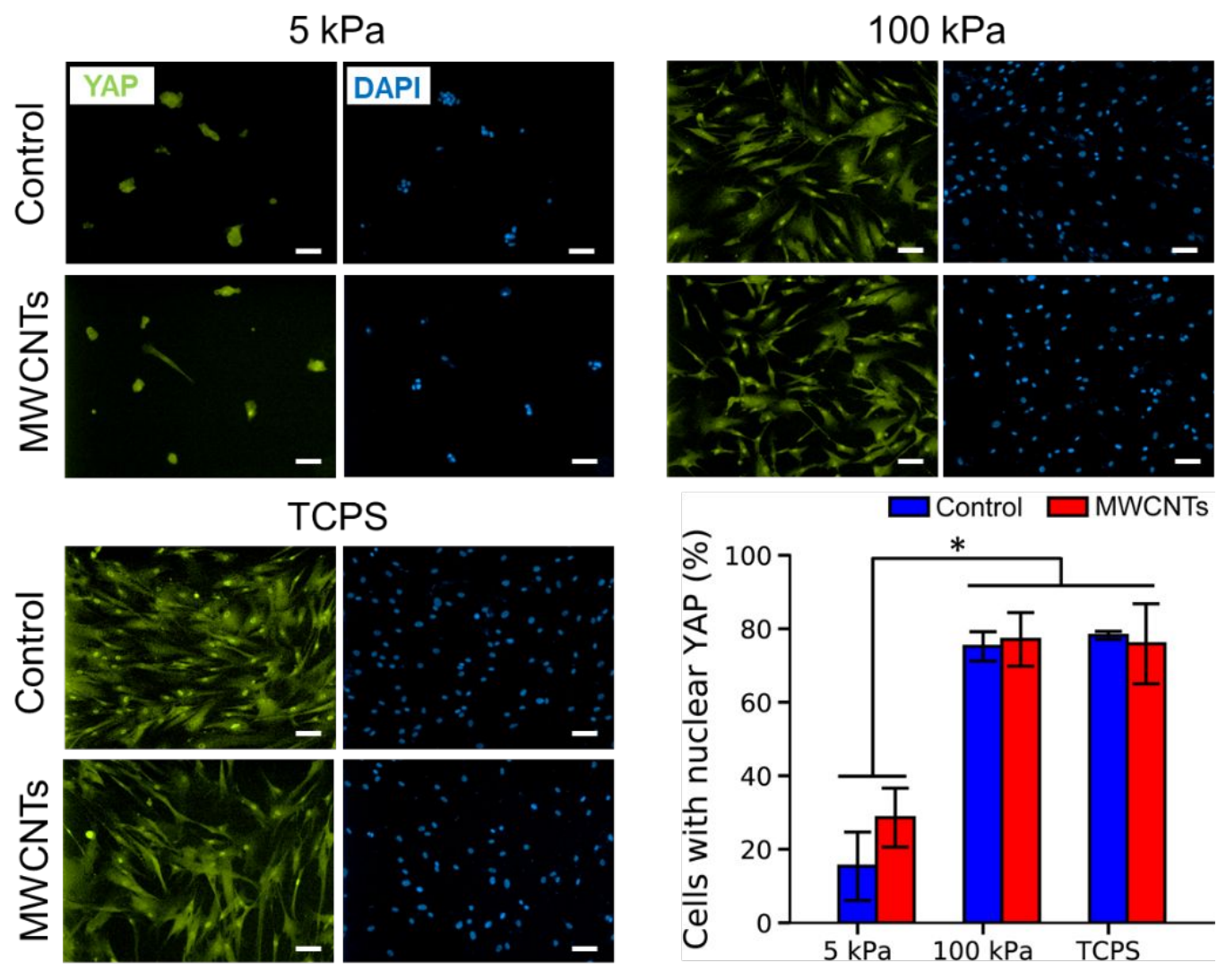

Figure S6: YAP intracellular localization in NHLFs on different substrates. Control: without MWCNTs treatment. MWCNTs: NHLFs were treated with MWCNTs for 3 days. * : significant difference $(p<0.05)$ between $3 \mathrm{kPa}$ group and $100 \mathrm{kPa}$, TCPS groups. Scale bars for all fluorescent images are $200 \mu \mathrm{m}$. Green: YAP; Blue: Nuclei. $\mathrm{n}>200$

\section{Time-stiffness superposition of MWCNT-induced fibrogenic responses of NHLFs}

Being analogous to the time-temperature superposition of polymer rheological data, the equivalent effects of MWCNTs treatment time and substrate stiffness on the fibrogenic responses of NHLFs to MWCNTs can be described by the proposed time-stiffness superposition principle. According to the principle, a set of MWCNTs-induced fibrogenic responses of the fibroblasts such as collagen I production measured on one substrate of stiffness $E$ can be shifted upon a reference stiffness $E_{0}$ as follows, 


$$
\log \alpha_{E}=\frac{-C_{1}\left(E-E_{0}\right)}{C_{2}+\left(E-E_{0}\right)}
$$

where $C_{1}$ and $C_{2}$ are constants, and $\alpha_{E}$ is the shift factor that horizontally shifts a given set of response with respect to the substrate stiffness. Equation (1) is derived from the Williams-Landel-Ferry (WLF) equation ${ }^{6}$.

Flow chart S1 illustrates the process from the normalization of fibrogenic response data to the determination of $\alpha_{E}, C_{1}$ and $C_{2}$ to the generation of the "master curve". The master curve enables the prediction of the fibrogenic response of interest at a specific stiffness over a broad time scale with the data sets measured on the substrates of several stiffnesses over the short periods of time. Briefly, the fibrogenic response data on the substrates of different stiffnesses are first normalized based on the reference substrate. For each substrate, an $\alpha_{E}$ will be estimated (for the reference substrate, $\alpha_{E}=1$ ) and used to shift the measured response from the time $t$ to $t^{\prime}=t \alpha_{E}$. Next, the response data $(F)$ will be plotted as a function of $t^{\prime}$ and fitted by a natural logarithmic function $F=a \ln t^{\prime}+b$, where $a$ and $b$ are constants. The $\alpha_{E}$ will be adjusted till the coefficient of determination $R_{1}^{2}$ is close to 1 . Moreover, the $\alpha_{E}$ should fit in Equation (1), which can be rearranged as follows,

$$
\frac{1}{\log \alpha_{E}}=-\frac{1}{C_{1}}-\frac{C_{2} 1}{C_{1} E-E_{0}}
$$

According to Equation (2), $\frac{1}{\log \alpha_{E}}$ is plotted as a function of $\frac{1}{E-E_{0}}$, and a linear regression is expected with the coefficient of determination $R_{2}^{2}$ close to 1 . If any of the coefficient of determination is significantly deviated from 1 , the shift factors will be adjusted accordingly. When both coefficients are close to 1 , the shift factors $\alpha_{E} \mathrm{~s}$ and constants $C_{1}$ and $C_{2}$ are determined. Subsequently, the master curve of fibrogenic responses as a function of time on the substrate of the reference stiffness is generated. 


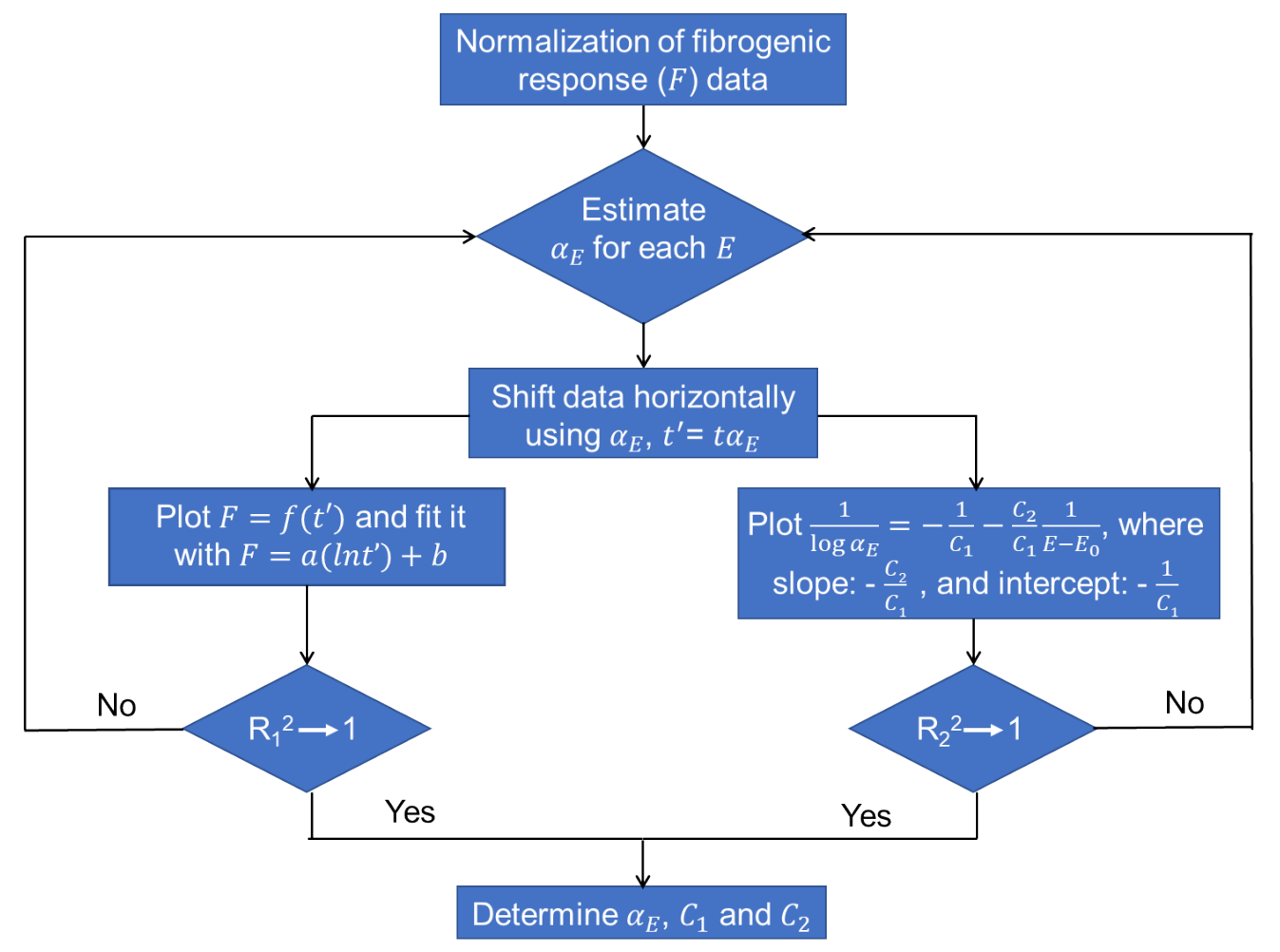

Chart S1. Time-stiffness superposition process.

The process is explained by taking example of collagen I ( $\mathrm{Col} \mathrm{I})$ production as the fibrogenic response. The collagen I production on the $5 \mathrm{kPa}$ PAAm gel of $E_{0}=4.9 \mathrm{kPa}$ is taken as the reference, and then the measurement on the different substrates is normalized to the value on $E_{0}=4.9 \mathrm{kPa}$ at Day 2 as given in Table S3.

Table S3. Collection and processes of fibrogenic data

\begin{tabular}{|c|c|c|c|c|c|c|c|c|}
\hline Substrate & \multicolumn{2}{|c|}{$5 \mathrm{kPa}$} & \multicolumn{2}{|c|}{$21 \mathrm{kPa}$} & \multicolumn{2}{|c|}{$100 \mathrm{kPa}$} & \multicolumn{2}{|c|}{ TCPS } \\
\hline Stiffness $E, \mathrm{kPa}$ & \multicolumn{2}{|c|}{4.9} & \multicolumn{2}{|c|}{21.2} & \multicolumn{2}{|c|}{100.8} & \multicolumn{2}{|c|}{2300000} \\
\hline Time $t$, day & 2 & 3 & 2 & 3 & 2 & 3 & 2 & 3 \\
\hline Col I expression, a.u. & 0.177 & 0.349 & 0.362 & 0.527 & 0.698 & 0.806 & 1.161 & 1.352 \\
\hline Normalized Col I expression, a.u. & 1 & 1.975 & 2.053 & 2.986 & 3.955 & 4.567 & 6.580 & 7.657 \\
\hline estimated $\alpha_{E}$ & \multicolumn{2}{|c|}{1} & \multicolumn{2}{|c|}{2} & \multicolumn{2}{|c|}{3} & \multicolumn{2}{|c|}{4} \\
\hline Shifted time $t^{\prime}$, day & 2 & 3 & 4 & 6 & 6 & 9 & 8 & 12 \\
\hline final $\alpha_{E}$ & & & \multicolumn{2}{|c|}{1.509} & \multicolumn{2}{|c|}{3.592} & \multicolumn{2}{|c|}{8.641} \\
\hline Shifted time $t^{\prime}$, day & 2 & 3 & 3.017 & 4.526 & 6.570 & 9.855 & 17.282 & 25.923 \\
\hline
\end{tabular}

For each stiffness an $\alpha_{E}$ is estimated and used to shift the time $t$ to $t^{\prime}=t \alpha_{E}$; for the reference $E_{0}, \alpha_{E}$

$=1$. With the new set of data, normalized Col I production is plotted as the function of $t^{\prime}$, and a natural 
logarithmic function $F=a \ln t^{\prime}+b$ is used to fit the shifted curve with the coefficient of determination $R_{1}^{2}$ (Figure S7), where $a$ and $b$ are constants. Meanwhile, the data are processed (as shown in Table S4) to plot $\frac{1}{\log \alpha_{E}}$ as a function of $\frac{1}{E-E_{0}}$ (Figure S8). The resulted linear function has the slope (S), intercept (I), and the coefficient $R_{2}^{2}$.

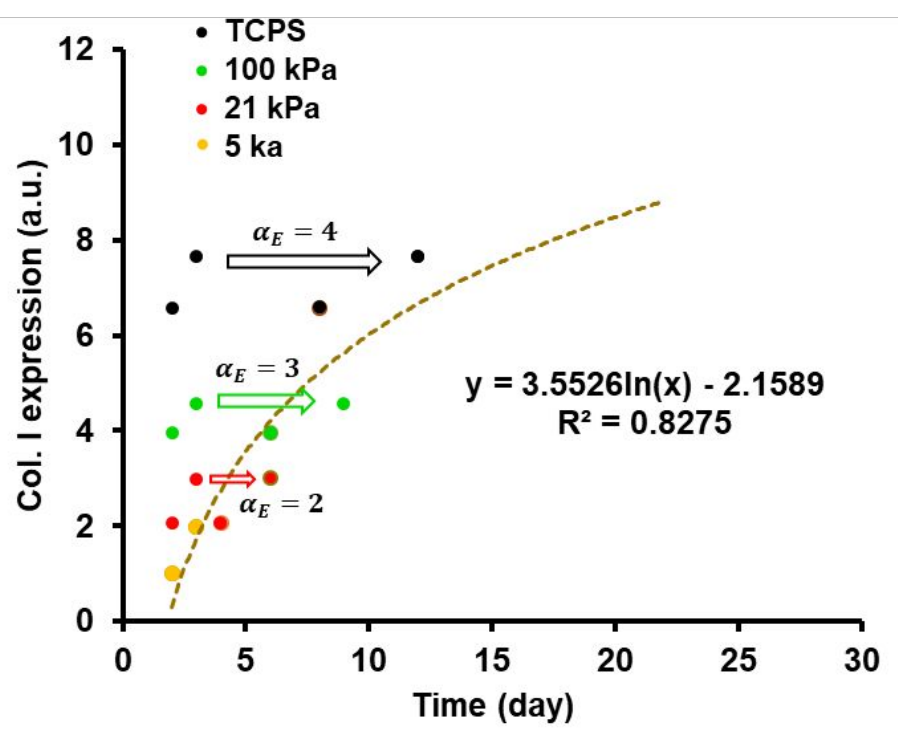

Figure S7. A master curve based on estimated $\alpha_{E} \mathrm{~S}$ referring to the $5 \mathrm{kPa}$ gel.

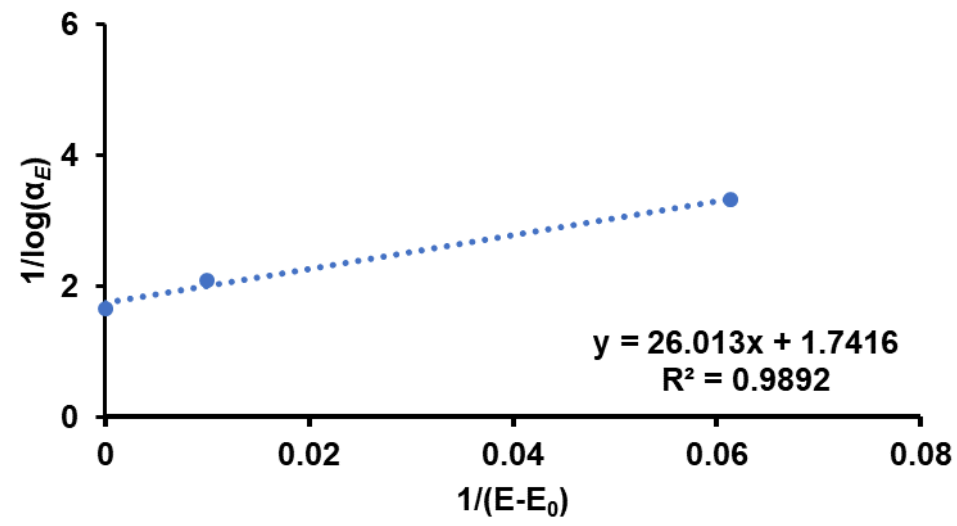

Figure S8. Linear plot of $\frac{1}{\log \alpha_{E}}$ as a function of $\frac{1}{E-E_{0}}$ based on estimated $\alpha_{E} \mathrm{~S}$ referring to the $5 \mathrm{kPa}$ gel.

Table S4. Data for plotting Equation (2) with the estimated and final $\alpha_{E} \mathrm{~S}$ 


\begin{tabular}{|c|c|c|c|c|c|c|c|}
\hline$E, \mathrm{kPa}$ & $\mathbf{1} /\left(\mathrm{E}-E_{0}\right)$ & estimated $\alpha_{\mathrm{E}}$ & $\log \left(\alpha_{\mathrm{E}}\right)$ & $\mathbf{1} / \log \left(\alpha_{\mathrm{E}}\right)$ & final $\alpha_{\mathrm{E}}$ & $\log \left(\alpha_{\mathrm{E}}\right)$ & $\mathbf{1} / \log \left(\alpha_{\mathrm{E}}\right)$ \\
\hline \hline 4.9 & & 1 & 0 & & & 0 & \\
\hline 21.2 & $6.13 \mathrm{E}-02$ & 2 & 0.301 & 3.322 & 1.509 & 0.179 & 5.600 \\
\hline 100.8 & $9.92 \mathrm{E}-03$ & 3 & 0.477 & 2.096 & 3.592 & 0.517 & 1.936 \\
\hline $2.30 \mathrm{E}+06$ & $4.35 \mathrm{E}-07$ & 4 & 0.602 & 1.661 & 8.641 & 0.937 & 1.068 \\
\hline
\end{tabular}

The $\alpha_{E}$ for each substrate except the reference is iterated till the two coefficients of determination $R_{1}^{2}$ and $R_{2}^{2}$ satisfy the criteria, for instance, both coefficients are smaller than 0.99 in this case. With the shift factors $\alpha_{E} \mathrm{~S}$ determined (Table S4), the "master curve" referring to $E_{0}$ $=4.9 \mathrm{kPa}$ is generated (Figure S9), and the linear plot related to Equation (2) is shown in Figure S10. The constants $C_{1}$ and $C_{2}$ are determined by the slope (S) and intercept (I) as $C_{1}=\frac{1}{I}$ and $C_{2}=\frac{S}{I}$, respectively.

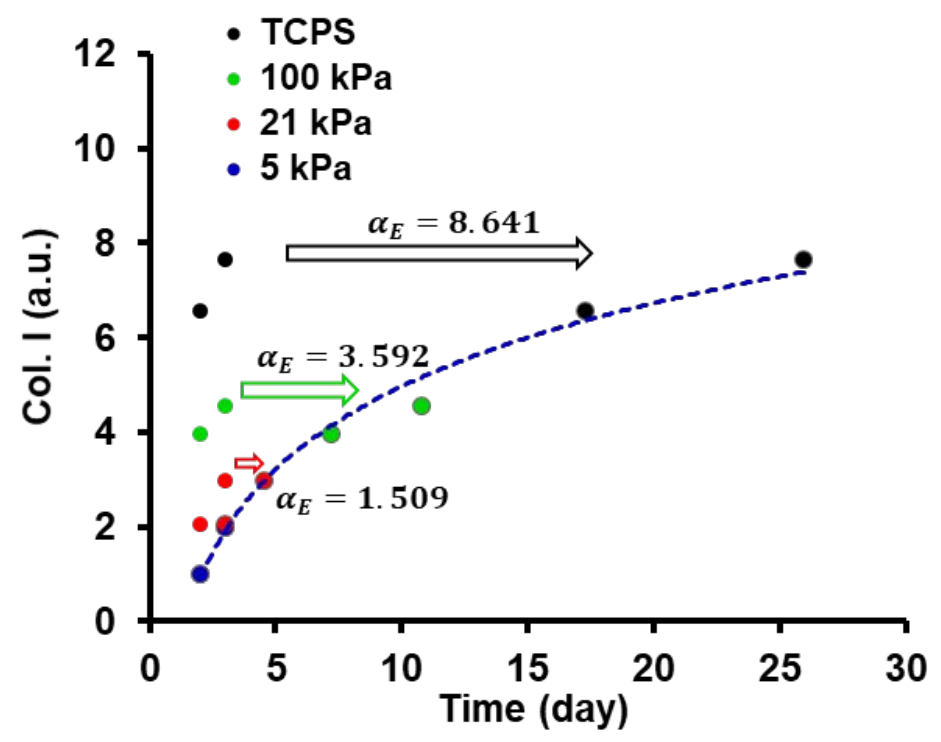

Figure S9. A master curve based on optimized $\alpha_{E} \mathrm{~S}$ referring to the $5 \mathrm{kPa}$ gel (same as the Figure 5e). 


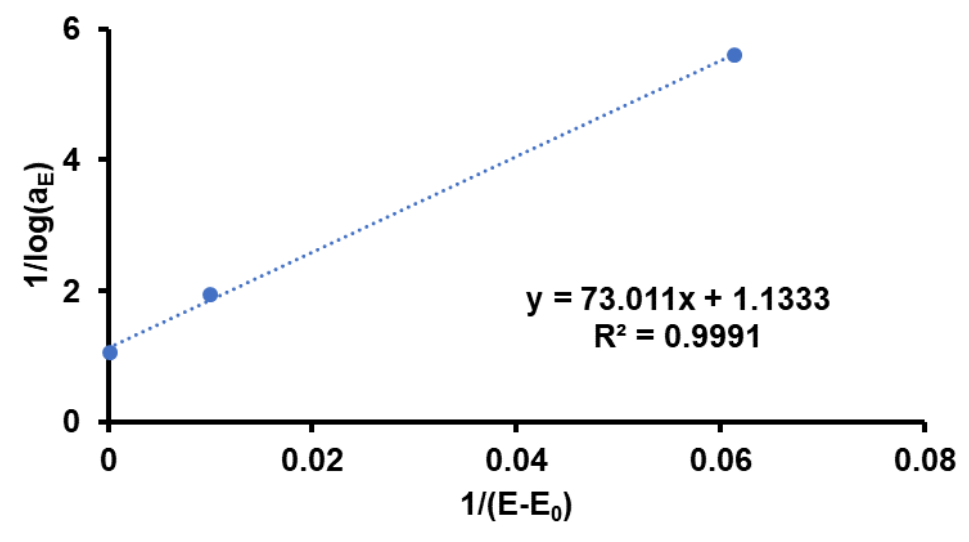

Figure S10. Linear plot of $\frac{1}{\log \alpha_{E}}$ as a function of $\frac{1}{E-E_{0}}$ based on optimized $\alpha_{E}$ S.

\section{Reference:}

1. Wang, Y.-L.; Pelham, R. J., [39] Preparation of a Flexible, Porous Polyacrylamide Substrate for Mechanical Studies of Cultured Cells. In Methods Enzymol., Academic Press: 1998; Vol. 298, pp 489-496.

2. Fischer, R. S.; Myers, K. A.; Gardel, M. L.; Waterman, C. M., Stiffness-Controlled ThreeDimensional Extracellular Matrices for High-Resolution Imaging of Cell Behavior. Nat. Protoc. 2012, 7 (11), 2056.

3. Linthicum, W.; Thanh, M.-T.; Vitolo, M.; Wen, Q., Effects of Pten Loss and Activated Kras Overexpression on Mechanical Properties of Breast Epithelial Cells. Int. J. Mol. Sci. 2018, 19 (6), 1613.

4. Wang, K.; He, X.; Linthicum, W.; Mezan, R.; Wang, L.; Rojanasakul, Y.; Wen, Q.; Yang, Y., Carbon Nanotubes Induced Fibrogenesis on Nanostructured Substrates. Environ. Sci. Nano 2017, 4 (3), 689-699.

5. Hirano, S.; Fujitani, Y.; Furuyama, A.; Kanno, S., Uptake and Cytotoxic Effects of Multi-Walled Carbon Nanotubes in Human Bronchial Epithelial Cells. Toxicol. Appl. Pharmacol. 2010, 249 (1), 8-15.

6. Williams, M. L.; Landel, R. F.; Ferry, J. D., The Temperature Dependence of Relaxation Mechanisms in Amorphous Polymers and Other Glass-Forming Liquids. J. Am. Chem. Soc. 1955, 77 (14), 3701-3707. 